\title{
NEEDLE ASPIRATION IN PERITONSILLAR ABSCESS: OUR EXPERIENCE IN CHITWAN
}

Tilak Raj Limbu' ${ }^{1,}$, Sovit Jung Baral', Amit Jha', Donjon Lamichane'

${ }^{1}$ Department of Otorhinolaryngology and HNS, Chitwan Medical College, Chitwan, Nepal

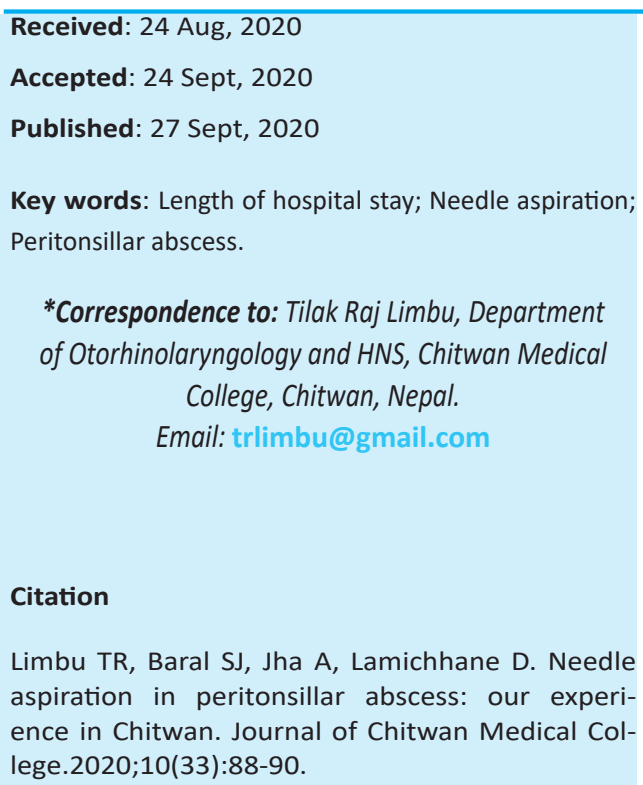

\begin{abstract}
Background: Peritonsillar abscess is one of deep neck space having significant morbidity and mortality. The treatment is still controversial regarding best method to perform. The aim of the study was to study the effect of needle aspiration in the treatment of peritonsillar abscess among the patients of Chitwan Medical College.
\end{abstract}

Methods: A retrospective study was designed which included all aged patients and were admitted in tertiary centre for peritonsillar abscess treatment between January 2015 to December 2019. All patient was treated with needle aspiration method under local spray anaesthesia and intravenous antibiotics. Number of aspirations, hospital stay, recurrence and complications were assessed as main outcomes. Descriptive analysis was performed by using SPSS version 16.0.

Results: Over 5-year duration, 57 patients were admitted for PTA and included for analysis. About $45(78.9 \%)$ of patients were under 40 years. Male dominance of $41(71.6 \%)$ was found. All cases were unilateral and no significant laterality found. All patients had prior medications outside before hospital visit. Length of hospital stay was 3.11+/-.939 days. Single or repeat aspiration of pus showed significant improvement of symptoms. One patient had recurrence and another one case had developed complication.

Conclusions: Our study showed needle aspiration is effective modality for peritonsillar abscess treatment due to early clinical improvement, low recurrence and decrease hospital stay.

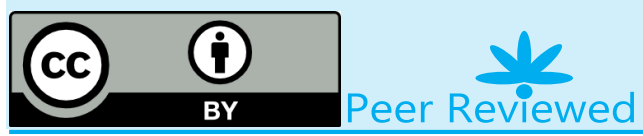

\section{INTRODUCTION}

Peritonsillar abscess (PTA), also known as Quinsy, is one of neck space abscess where pus is collected between tonsillar capsule and superior constrictor muscle, causes severe morbidity, even fatal if neglected. Due to severity of symptoms like odynophagia, trismus, fever, patients visit emergency as well as ENT department for treatment. The incidence of PTA is 10-37 per 100,000 population with more prevalence in young adult. ${ }^{1}$

Most accepted theory of abscess formation is secondary to tonsillitis and presence of Weber's gland. Due to presence of mixed flora, there is almost common acceptance of using parenteral broad-spectrum antibiotics. Incision and drainage (I \& D) and Needle aspiration are two modalities of treatment more accepted, which is followed by intravenous antibiotics having almost same success rate. Treatment modalities differs with different institute's protocol. Needle aspiration is quicker, less painful, easy to perform with less morbidity while incision and drainage has benefit of less recurrence and short hospital stay. Several literatures are found comparing both management procedures showing almost same result. Studies have reported no significance differences between and incision regarding different outcomes like length of stay, resumption of normal diet, recurrence rate, ${ }^{2-4}$ while few studies have reported in favour of aspiration..$^{5-7}$ However, Mansour et al has concluded incision has good result. ${ }^{8}$ The aim was to study the effect of needle aspiration in the treatment of peritonsillar abscess among the patients of Chitwan Medical College.

\section{METHODS}

A retrospective study was conducted in Department of Ear Nose Throat, Chitwan Medical College, Chitwan, Nepal from January 2015 to December 2019. Institutional ethical clearance was taken. All necessary data were collected from hospital medical records who were hospitalized for confirmed peritonsillar abscess in ENT ward.

All aged patients were included in this study who needed hospital admission, most of time oral treatment was not possible. The evolution time of symptoms, presence of fever, odynophagia, sore throat, trismus was listed. Patient profile with time of admission and discharge as well as medication history prior to hospitalisation was recorded.

Initially a needle aspiration was performed for each clinically diagnosed peritonsillar abscess patient to confirm the diagno- 
sis for purulent secretion, to locate the abscess cavity. A wide bore $18 \mathrm{G}$ needle with $10 \mathrm{ml}$ syringe was inserted over the most bulging area or just outside the meeting point of anterior tonsillar pillar line and uvula base after using 10\% lidocaine spray. The diagnosis of peritonsillar abscess was not confirmed if the needle aspiration remained negative after one or two aspirations and was excluded from study. If aspiration of pus was positive. further additional needle insertions in different locations may be performed in a single treatment procedure. All cases were started immediately with intravenous antibiotics, analgesics and intravenous fluids after admission. Routine haematological investigation was sent.

Absence of fever, start of oral intake, trismus improvement, pain improvement and decreased local swelling were considered clinical improvement cure. Length of stay was chosen as a primary outcome as it is one of best indicators of clinical recovery and success of treatment. Secondary outcomes included the need of re-aspiration, complication of disease and recurrence. Patients were discharged on oral antibiotics with advice of follow up after 7 days. The data was collected and entered in Microsoft Excel and then transferred into SPSS version 16. Descriptive statistical analysis was performed.

\section{RESULTS}

Table 1: Socio-demographic and clinical characteristics of peritonsillar abscess

\begin{tabular}{|c|c|c|}
\hline Parameters & & Frequency (\%) \\
\hline \multirow{5}{*}{ Age groups (years) } & 11 to 20 & $18(31.6)$ \\
\hline & $21-30$ & $19(33.3)$ \\
\hline & $31-40$ & $8(14.0)$ \\
\hline & $41-50$ & $7(12.3)$ \\
\hline & $51-60$ & $5(8.8)$ \\
\hline \multirow{2}{*}{ Sex } & Male & $41(71.6)$ \\
\hline & Female & $16(28.1)$ \\
\hline \multirow{2}{*}{ Side } & Right & $27(47.4)$ \\
\hline & Left & $30(52.6)$ \\
\hline \multirow{3}{*}{ Onset of symptoms(days) } & 2 & $22(38.6)$ \\
\hline & 3 & $29(50.9)$ \\
\hline & 4 & $6(10.5)$ \\
\hline Medication before admission & Yes & $57(100)$ \\
\hline \multirow{3}{*}{ No of aspiration } & $1^{\text {st }}$ & $23(40.4)$ \\
\hline & $2^{\text {nd }}$ & $33(57.9)$ \\
\hline & $3^{\text {rd }}$ & $1(1.8)$ \\
\hline \multirow{5}{*}{ Duration of stay(days) } & 1 & $1(1.8)$ \\
\hline & 2 & $15(26.3)$ \\
\hline & 3 & $22(38.6)$ \\
\hline & 4 & $15(26.3)$ \\
\hline & 5 & $4(7.0)$ \\
\hline \multirow{2}{*}{ Difficulty in food intake } & Yes & $55(96.5)$ \\
\hline & No & $2(3.5)$ \\
\hline \multirow{2}{*}{ Trismus } & Yes & $33(57.9)$ \\
\hline & No & $24(42.1)$ \\
\hline \multirow{2}{*}{ Complications } & Yes & $1(1.8)$ \\
\hline & No & $56(98.2)$ \\
\hline \multirow{2}{*}{ Recurrence } & Yes & $1(1.8)$ \\
\hline & No & $56(98.2)$ \\
\hline
\end{tabular}

Total 57 patients were included in this study. Out of them, 41 were male and 16 females. About $45(78.9 \%)$ of patients were below 40 years in this study. Out of 57 patients, 30(52.6\%) patients had peritonsillar abscess on left side and right-side involvement was seen in 27 patients (47.4\%).

All patients included in the study had some type of medications before hospital visit. While 29 (50.9\%) patients had ENT consultation on after 3 days of onset of symptoms, $22(38.6 \%), 6(10.5 \%)$ patients consulted after 2 and 4 days respectively. In this study, $23(40.4 \%)$ required single needle aspiration, $33(57.9 \%)$ second aspiration and only $1(1.8 \%)$ had third aspiration. About 55(96.5\%) of patients had difficulty of swallowing at the time of hospital visit and only 33(57.9\%) patients had trismus of varying degree. One patient $(1.8 \%)$ had complication. Recurrence was seen in one patient(1.8\%) (Table 1).

\section{DISCUSSION}

Peritonsillar abscess is the commonest entity among deep neck space abscess. ${ }^{9}$ It affects people of all age groups with peak in the second and third decades of life. In our study majority (79\%) of patients were aged under 40 years. ${ }^{1}$

Most of PTA are unilateral in nature but may vary on laterality and gender basis. Dalton et al has reported incidence of bilateral PTA of $4.9 .{ }^{10}$ All of our cases had unilateral abscess with laterality to left side $52.6 \%$ with male predominance of $72 \% .{ }^{11-13}$

Patients with PTA generally visit hospital with the chief complaint of difficulty in swallowing and trismus, fever. ${ }^{14}$ In our study 55 (96.5\%) had difficulty in swallowing while 33 (57.9\%) had trismus. Majority of patients had given history of fever but afebrile during hospital visit, so fever status was not evaluated. This condition is probably due to antipyretic use before hospital visit.

Nearly half of the cases (29 out of 57) had consulted our ENT OPD after 3 days of onset of symptoms. All of them had medications (oral antibiotics, analgesic, antipyretics oral gargle) from local pharmacy prior to hospital visit. This is probably due to self-medication habit in this region which cannot be ignored and requires further study. Lepelletier et al stated majority of patients (92\%) had consulted within 10 days before admission. ${ }^{15}$

Mainly two modalities of pus drainage is popular among Otolaryngologists, incision \& drainage (I\&D) and needle aspiration. Powell et al stated that overall there is no convincing evidence in favour of aspiration or incision and drainage, having recurrence of $9-22 \% .{ }^{16}$ However, the use of needle aspiration as primary treatment modality had also been reported. ${ }^{17}$

King first described use of wide bore needle Needle1961. ${ }^{18}$ Needle aspiration is quick and easy to perform procedure with additional advantages of repetition, less painful. Single setting of pus aspiration may be enough for diagnosis, treatment and culture \& sensitivity sampling. It also known to have less com- 
plications.

The effectiveness of needle aspiration as definitive treatment of peritonsillar abscess has also been reported in literatures. A study by Bhat et al had shown $90 \%$ improvement after $1^{\text {st }}$ aspirations. ${ }^{5}$ Our study shows $23(40.4 \%)$ of patients improved on $1^{\text {st }}$ and $33(57.9 \%)$ on $2^{\text {nd }}$ aspiration. Second aspiration was performed on same sitting or next ward visit. Continuous bulging of superior peritonsillar area and/or throat discomfort was indicated for additional aspiration. Only $1(1.8 \%)$ patient needed $3^{\text {rd }}$ aspiration. ${ }^{12}$

Length of hospital stay in our study was 1-5 days, mean of 3.11+/- 0.939 days. This was supported by Mansour et 2019 which showed length of hospital stay in aspiration group of 1-7 days with mean 3 days. ${ }^{8,12}$ Length of hospital stay depended on the basis clinical outcomes -improvement in the oral intake, absence of trismus and afebrile status in our study.

In our study, one patient had recurrence after 7 days of discharge, which was less than stated in other study. ${ }^{16}$ The patient was readmitted and same procedure repeated and was cured on 5th day. One patient young adult male had developed complication of parapharyngeal and post triangle neck cellulitis during hospital stay, which had prolonged IV antibiotics and was cured. Study by Klug et al have shown that complications can be seen in patients (male,>40 years) who are treated surgically and is under medication. ${ }^{19}$

The study is not devoid of limitations. The first limitation was the retrospective nature of the study and the data was obtained from medical records. There can be loss of data owing to the fact we had small sample size in this study.

\section{CONCLUSION}

Our study confirmed that needle aspiration is effective primary treatment modality for treatment of peritonsillar abscess. It is easy to perform with less complications and short hospital stay reduces financial burden. Further study with large sample size is recommended.

\section{CONFLICT OF INTEREST: None}

FINANCIAL DISCLOSURE: None

\section{REFERENCES:}

1. Sowerby LJ, Hussain Z, Husein M. The epidemiology, antibiotic resistance and post-discharge course of peritonsillar abscesses in London, Ontario. Journal of Otolaryngology-Head \& Neck Surgery. 2013;42(1):5. [DOI]

2. Maharaj D, Rajah V, Hemsley S. Management of peritonsillar abscess. The Journal of Laryngology \& Otology. 1991;105(9):743-5. [DOI]

3. Schechter GL, Sly DE, Roper AL, Jackson RT. Changing face of treatment of peritonsillar abscess. The Laryngoscope. 1982;92(6):657-9. https://doi. org/10.1002/lary.1982.92.6.657

4. Spires JR, Owens JJ, Woodson GE, Miller RH. Treatment of peritonsillar abscess: a prospective study of aspiration vs incision and drainage. Archives of Otolaryngology-Head \& Neck Surgery. 1987;113(9):984-6. [DOI]

5. Bhat VS, A A. Role of Needle Aspiration in the Management of Peritonsillar Abscess. Sch J App Med Sci. 2016;4(7E):2600-2. [DOI]

6. Herzon FS. Peritonsillar abscess: incidence, current management practices, and a proposal for treatment guidelines. The Laryngoscope. 1995;105(S3):1-17. [DOI]

7. Johnson RF, Stewart MG, Wright CC. An evidence-based review of the treatment of peritonsillar abscess. Otolaryngology-Head and Neck Surgery. 2003;128(3):332-43. [DOI]

8. Mansour C, De Bonnecaze G, Mouchon E, Gallini A, Vergez S, Serrano E. Comparison of needle aspiration versus incision and drainage under local anaesthesia for the initial treatment of peritonsillar abscess. European Archives of Oto-Rhino-Laryngology. 2019;276(9):2595-601. [DOI]

9. Maharjan S, Joshi R, Rijal A, Dhungana A, Shrestha K. Pattern of deep neck space infections at a tertiary hospital, Kathmandu, Nepal. Nepal Medical College Journal. 2020;22(1-2):44-8. [DOI]

10. Dalton RE, Abedi E, Sismanis A. Bilateral peritonsillar abscesses and quinsy tonsillectomy. Journal of the National Medical Association.
1985;77(10):807. [PMID]

11. Freire G, Dos Santos J, Rolón P, Pinheiro G, Sampaio A. Peritonsillar abscess: epidemiology and relationship with climate variations. The Journal of Laryngology and Otology. 2017;131(7):627. [DOI]

12. Shaul C, Koslowsky B, Rodriguez M, Schwarz Y, Muahnna N, Peleg U, et al. Is needle aspiration for peritonsillar abscess still as good as we think? A long-term follow-up. Annals of Otology, Rhinology \& Laryngology. 2015;124(4):299-304. [DOI]

13. Slouka D, Hanakova J, Kostlivy T, Skopek P, Kubec V, Babuska V, et al. Epidemiological and Microbiological Aspects of the Peritonsillar Abscess. International Journal of Environmental Research and Public Health 2020;17(11):4020. [DOI]

14. Souza DL, Cabrera D, Gilani WI, Campbell RL, Carlson ML, Lohse CM, et al. Comparison of medical versus surgical management of peritonsillar abscess: a retrospective observational study. The Laryngoscope. 2016;126(7):1529-34. [DOI]

15. Lepelletier D, Pinaud V, Le Conte P, Bourigault C, Asseray N, Ballereau $F$, et al. Peritonsillar abscess (PTA): clinical characteristics, microbiology, drug exposures and outcomes of a large multicenter cohort survey of 412 patients hospitalized in 13 French university hospitals. European Journal of Clinical Microbiology \& Infectious Diseases. 2016;35(5):867-73. [DOI]

16. Powell J, Wilson J. An evidence-based review of peritonsillar abscess Clinical otolaryngology. 2012;37(2):136-45. [DOI]

17. Mehanna H, Al-Bahnasawi L, White A. National audit of the management of peritonsillar abscess. Postgraduate medical journal. 2002;78(923):5457. [DOI]

18. King J. Aspiration treatment of peritonsillar abscess. Journal of the Medical Association of Georgia. 1961;50:18-9. [PMID]

19. Klug TE, Greve T, Hentze M. Complications of peritonsillar abscess. Annals of clinical microbiology and antimicrobials. 2020;19(1):1-17. [DOI] 